\title{
Genotypic and phenotypic spectrum of CCDC141 variants in a Chinese cohort with congenital hypogonadotropic hypogonadism
}

\author{
Qiao Hou 1,2,3,4, Jiayu Wu11,2,3, Yaguang Zhao 1,2,3, Xinying Wang 1,2,3, Fang Jiang 1,2,3, Dan-Na Chen ${ }^{5}$, Ruizhi Zheng \\ Meichao Men $\mathbb{1}^{7}$ and Jia-Da Li' ${ }^{1,2,3}$
}

${ }^{1}$ School of Life Sciences, Central South University, Changsha, Hunan, China, ${ }^{2}$ Hunan Key Laboratory of Medical Genetics, Central South University, Changsha, Hunan, China, ${ }^{3}$ Hunan Key Laboratory of Animal Models for Human Diseases, Central South University, Changsha, Hunan, China, ${ }^{4}$ Department of Rehabilitation Medicine, Xiangya Third Hospital, Central South University, Changsha, Hunan, China, ${ }^{5}$ Department of Basic Medical Sciences, Changsha Medical University, Changsha, Hunan, China, ${ }^{6}$ Department of Endocrinology, The People's Hospital of Henan Province, Zhengzhou, Henan, China, and ${ }^{7}$ Health Management Center, Xiangya Hospital, Central South University, Changsha, Hunan, China

\section{Correspondence} should be addressed to M Men or Jia-Da Li Email

menmeichao@csu.edu.cn or lijiada@sklmg.edu.cn

\begin{abstract}
Objective: To identify CCDC141 variants in a large Chinese cohort with congenital hypogonadotropic hypogonadism $(\mathrm{CHH})$ and to assess the contribution of $\mathrm{CCDC141}$ to $\mathrm{CHH}$.

Design: Detailed phenotyping was conducted in $\mathrm{CHH}$ patients with CCDC141 variants and co-segregation analysis was performed, when possible.

Methods: Whole-exome sequencing was performed in $177 \mathrm{CHH}$ patients and 450 unrelated, ethnically matched controls from China.

Results: Seven novel CCDC141 rare sequencing variants (RSVs) were identified in $12 \mathrm{CHH}$ pedigrees. Four of the variants were private mutations; however, p.Q409X, p.Q871X and p.G1488S were identified in more than one patient. Up to $75 \%$ (9/12) of patients had mutations in other $\mathrm{CHH}$-associated genes, which is significantly higher than $\mathrm{CHH}$ patients without CCDC141 RSVs. The co-segregation analysis for eight CHH families showed that 75\% (6/8) CCDC141 RSVs were inherited from their fertile parents. Over half $(58.3 \%, 8 / 18)$ of the patients exhibited other clinical deformities in addition to hypogonadism. One patient harbouring a CCDC141 RSV showed a reversal of $\mathrm{CHH}$ after sex-steroid replacement.

Conclusions: Our results broaden the genotypic spectrum of CCDC141 in CHH, as CCDC141 RSVs alone do not appear sufficient to cause $\mathrm{CHH}$. The phenotypic spectrum in patients with CCDC141 RSVs is much wider than originally believed.
\end{abstract}

\section{Introduction}

Congenital hypogonadotropic hypogonadism (CHH), due to a deficiency in the secretion of gonadotropinreleasing hormone, is a rare genetic disorder characterised by abnormal puberty and infertility and is frequently associated with non-reproductive phenotypes. Anosmia/ hyposmia (i.e. lack of or a defective sense of smell), termed Kallmann syndrome (KS) in $\mathrm{CHH}$ patients, has been observed to co-occur in $\sim 50 \%$ of them, with the remaining being normosmic (nCHH) (1). Other phenotypes associated with $\mathrm{CHH}$ include synkinesia, cerebellar ataxia, hearing loss, eye movement disorders, renal aplasia, high arched palate, labial or palatine cleft, (c) 2020 European Society of Endocrinology Printed in Great Britain
Published by Bioscientifica Ltd. 
skeletal anomalies, dental agenesis and cardiovascular defects $(2,3,4,5)$.

During embryonic development, GnRH neurons originating in the nasal placode will migrate along the olfactory axons towards the CNS. Upon arriving at the CNS, olfactory axons will sneak into the olfactory bulb, whereas GnRH neurons will migrate caudally on a bundle of non-sensory olfactory axons towards the hypothalamus (6). Pathological changes in any of these processes can lead to $\mathrm{CHH}$. To date, over 30 genes have been found to be associated with $\mathrm{CHH}$ and many encode receptor-ligand pairs, such as FGFR1 (OMIM 136350) and FGF8 (OMIM 600483), GNRHR (OMIM 138850) and GNRH1 (OMIM 152760), TACR3 (OMIM 162332) and TAC3 (OMIM 162330), KISS1R (OMIM 604161) and KISS1 (OMIM 603286), as well as PROKR2 (OMIM 607123) and PROK2 (OMIM 607002) (7, 8, 9, 10, 11, 12, 13). Furthermore, disruption in the dynamic remodelling of the cell's cytoskeleton during migration of GnRH neurons may also result in $\mathrm{CHH}(14,15,16)$. Recently, Topaloglu et al. reported that the cytoskeletal protein, CCDC141, is expressed in GnRH neurons as well as olfactory fibres and that its knockdown reduced migration of $\mathrm{GnRH}$ neurons (17). They also identified inactivating CCDC141 variants in $3.3 \%$ of $\mathrm{CHH}$ individuals (18); however, the contribution of CCDC141 to $\mathrm{CHH}$, in particular, has not been reported in other cohorts.

Our objective in this study was to identify CCDC141 variants in a Chinese $\mathrm{CHH}$ cohort of 177 patients and to assess the contribution of CCDC141 to $\mathrm{CHH}$.

\section{Subjects and methods}

\section{Subjects and clinical evaluation}

This study consisted of 177 unrelated patients (146 males, mean age at diagnosis: 21.9 years), who were previously diagnosed with $\mathrm{CHH}$ based on (1) failure to enter spontaneous puberty by age 18 or through medically induced puberty before this age, (2) low sex-steroid levels in association with inappropriately low/normal gonadotropin levels and an abnormal/normal response to a GnRH stimulation test, (3) exclusion from other anterior pituitary dysfunctions, (4) normal radiological imaging of the hypothalamic and pituitary areas, and (5) for KS, anosmia/hyposmia according to formal testing (UPSIT score $<5$ th percentile by age; University of Pennsylvania Smell Identification Test). All patients (112 with KS and 65 with $\mathrm{nCHH}$ ) were recruited from the People's Hospital of Henan Province (Zhengzhou, China) and Xiangya Hospital (Changsha, China).

The brain and olfactory bulbs were examined using MRI, when possible. Bone densitometry, body weight, skin texture, height, limb development, tooth development, testis size, sperm count and medication history were collected and analysed retrospectively. The patients or their adult parents signed an informed consent form. A group of 450 unrelated, ethnically matched individuals served as the control. The study was approved by the ethics committee of the School of Life Sciences, Central South University (No. 2017030801).

\section{Whole-exome sequencing and co-segregation analysis}

Genetic testing was performed by whole-exome sequencing (WES) according to previously outlined methods (19). Briefly, genomic DNA was fragmented and 180-300 bp products were chosen. Exons and flanking intronic regions were captured by Agilent SureSelect Human All Exon V6 enrichment platform (Agilent Technologies) and subsequently sequenced on Illumina HiSeq 4000 platform (Illumina, San Diego, CA). The average sequencing depth was of $50 \times$. Copy number variations analysis was performed using ExomeDepth algorithm as described previously (20). We screened for rare sequencing variants (RSVs, <1\% in the dbSNP, ExAC, ESP6500, Genome Aggregation Database (gnomAD), Chinese Millionome Database (CMDB) and 1000 Genomes) in CCDC141 and a panel of genes involved in $\mathrm{CHH}$, including $A X L$, DUSP6, FEZF1, FGF8, FGF17, FGFR1, FLRT3, GNRH1, GNRHR, HESX1, HS6ST1, IL17RD, ANOS1, KISS1, KISS1R, NSMF, PROK2, PROKR2, SEMA3A, SEMA3E, PLXNA1, SEMA7A, SPRY4, TAC3, TACR3, WDR11, LEP, LEPR, NROB1, PCSK1, STUB1, CHD7, DMXL2, SOX2, PNPLA6, RNF216, SOX10 and $\operatorname{OTUD} 4(21,22,23)$. Variants were named according to the Human Genome Variation Society's nomenclature (24). The detected variants were verified by PCR-Sanger sequencing with special primers (Supplementary Table 1, see section on supplementary materials given at the end of this article). Co-segregation analysis was carried out on all available family members.

\section{Interpretation of RSVs}

Mutations were interpreted according to the guidelines by the American College of Medical Genetics and Genomics (ACMG) (25). Missense variants were automated in InterVar (http://wintervar.wglab.org/) and supplemented 
manually, including inputting information on phenotypes, inheritance and segregation. Variants were divided into five categories: benign (B), likely benign, uncertain significance (U), likely pathogenic(LP) and pathogenic (P). Furthermore, four commonly used in silico tools were utilised to predict the function of the detected variants, including Polyphen2, MutationTaster, SIFT and Combined Annotation Dependent Depletion. A variant was considered deleterious if it was predicted to be damaging by at least two of the in silico tools.

\section{Results}

\section{Phenotypic and genotypic summary of the CHH patients}

A total of 177 unrelated patients (146 men) were included in this study. Among them, 63.3\% (112/177) were diagnosed with $\mathrm{KS}$ and $36.7 \%$ (65/177) with $\mathrm{nCHH}$, which is comparable to other cohorts $(2,3,4,5)$ (Table 1). It should be noted that up to $24 \%$ of the males in this cohort presented with unilateral or bilateral cryptorchidism, whereas only 5.9\% males presented with cryptorchidism in a $\mathrm{CHH}$ cohort studied by Nair et al. (4). Additional pathologies were also identified in these patients, including congenital hearing loss $(9.7 \%)$, dental agenesis (11.8\%), skeletal anomalies (10.1\%), ocular defects (10.1\%), ataxia (2.8\%), cleft lip/palate $(1.7 \%)$, renal agenesis $(4 \%)$, mirror movement $(2.8 \%)$ and external ear defects (4\%). Furthermore, family histories of $\mathrm{CHH}$, delayed puberty or isolated congenital olfactory defects were identified in $21.9 \%$ (43/177) of patients. The occurrence rates of some of these pathologies within other $\mathrm{CHH} / \mathrm{KS}$ cohorts are listed in Table 1.

Exome sequencing was performed on $177 \mathrm{CHH}$ probands and $66.1 \%(117 / 177)$ of patients harboured deleterious RSVs in $34 \mathrm{CHH}$-associated genes (Fig. 1). The most frequently mutated gene was PROKR2 $(14.7 \%$ of probands), followed by CHD7 (10.2\%), ANOS1 (8.5\%) and FGFR1 (8.5\%). No mutation was identified in TAC3, HESX1, FLRT3, LEP and NROB1.

\section{CCDC141 RSVs were significantly enriched in $\mathrm{CHH}$ patients vs controls}

Seven heterozygous CCDC141 RSVs, including two protein truncating variants and five missense variants, were identified in 12 out of the $177 \mathrm{CHH}$ probands (Fig. 2). Only two missense RSVs (p.G1488S CCDC141 and
p.P7L CCDC141) were found in the controls. The allele frequency of CCDC141 RSVs was significantly higher in $\mathrm{CHH}$ patients compared to the controls $(6.8 \%, 12 / 177$ vs $0.2 \%, 2 / 450 ; P=0.000$, Fisher's exact test).

Four out of seven CCDC141 variants identified in the patients were private mutations (p.R123G, p.Q256K, p.R1384S and p.Q1407K); however, p.Q409X, p.Q871X and p.G1488S were identified in multiple unrelated patients. These variants were not found in the controls except p.G1488S CCDC141. The occurrence frequencies of p.R123G, p.Q409X, p.Q871X and p.G1488S variants were $0.00008,0.0002,0.000026$ and 0.0015 , respectively, in the gnomAD database, whereas the other RSVs were not detected in this database (Table 2). All RSVs were predicted to cause disease according to MutationTaster, and all variants except p.R1384S were predicted to be probably damaging or damaging according to PolyPhen-2 (Table 2). Per the ACMG guidelines, two variants (p.Q409X and p.Q871X) were pathogenic and the remaining were of uncertain significance (Table 2).

Nine out of 12 probands also carried variants in other CHH-associated genes, including PCSK1, ANOS1, PROKR2, AXL, SOX10, HS6ST1, PNPLA6 and FGFR1 (Table 2). The frequency of one patient carrying variants in at least two genes was significantly higher in the CCDC141 RSVs' positive group $(75 \%, 9 / 12)$ relative to the CCDC141 RSVs' negative group (31.4\%, 33/105; $P=0.008$, Fisher's exact test).

\section{Clinical characteristics of $\mathrm{CHH}$ patients harbouring CCDC141 RSVs}

Clinical data from the 12 probands with CCDC141 RSVs are summarised in Table 3 . The mean age at diagnosis was 22.6 years. Seven males were affected with KS, while five patients (three males and two females) had $\mathrm{nCHH}$. Cryptorchidism occurred in four KS patients and one nCHH patient. Eight patients exhibited non-reproductive clinical deformities in addition to hypogonadism. Dental agenesis was detected in three patients (probands 2, 4 and 11), mainly manifesting as small and missing teeth. Hypopigmentation was seen in proband 8, carrying p.Q871X CCDC141 and p.L80V SOX10 mutations. Neurological defects were also common in these patients. Colour weakness was found in probands 3 and II:4 in Family 2. Congenital high myopia was found in probands 4 and 10. Congenital anhidrosis was seen in proband 4. Delayed mental development was seen in proband 11 . 


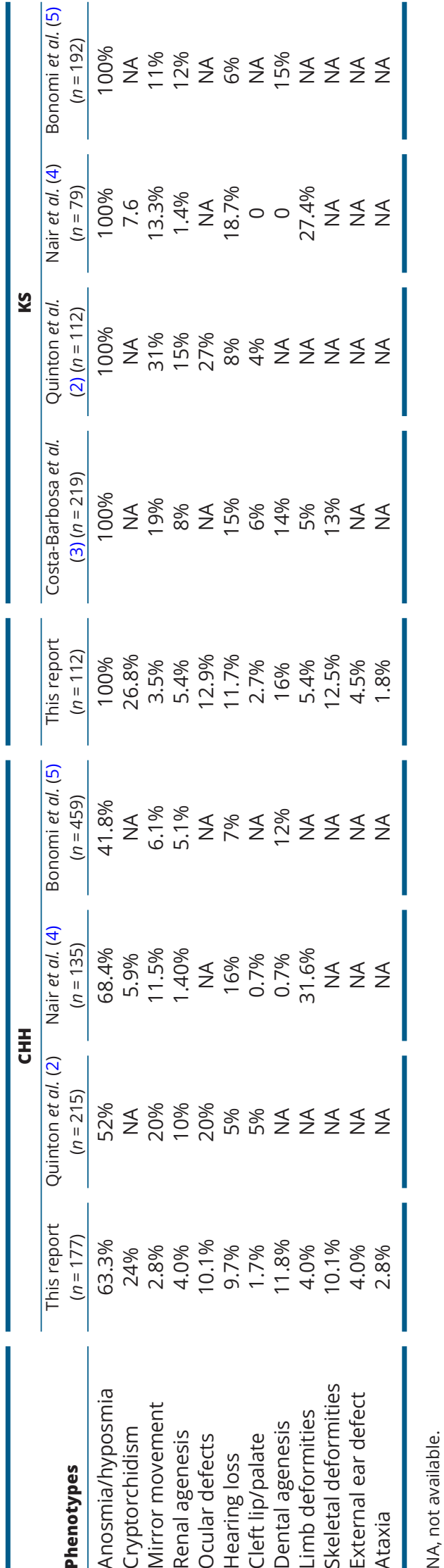

\section{Pedigree investigation}

We also analysed the variants in the available family members of eight probands (Fig. 3 and Table 3). Interestingly, six probands (1, 2, 3, 4, 5 and 11) inherited their CCDC141 RSVs from the unaffected parents, whereas probands 7 and 8 carried de novo CCDC141 RSVs.

In Family 1, the proband (III:7) was diagnosed as KS, carrying p.N355I DUSP6 and p.Q1407K CCDC141 variants. His brother (III:8), carrying p.N355I DUSP6, showed anosmia and his father (II:5), harbouring p.Q1407K CCDC141, was normal. In Family 2, the proband (III:2) inherited a p.R123G CCDC141 mutation from his father and a p.V162I PCSK1 mutation from his mother. The proband showed dental agenesis and ataxia in infancy, in addition to nCHH. Both parents showed normal puberty and were fertile. However, his father (II:4), carrying the p.R123G CCDC141 mutation, showed colour weakness. His mother (II:5), carrying the p.V162I PCSK1 mutation, showed sparse armpit hair. The maternal uncle (II:8), harbouring the p.V162I PCSK1 mutation, showed hypospadias and non-liquefaction of sperm, but displayed normal sexual characteristics and had a baby after one cycle of treatment. Interestingly, the maternal grandma (I:4), who also carried p.R123G CCDC141 and p.V162I PCSK1, manifested delayed puberty and no armpit or pubic hair, but showed normal fertility (Fig. 3 and Table 3).

In Family 3, the proband (II:2), carrying trigenic mutations of p.Q256K CCDC141, p.R270H PROKR2 and p.R617Q SEMA3A, showed left cryptorchidism and colour weakness in addition to $\mathrm{nCHH}$. His mother (I:2), carrying two mutations of p.R270H PROKR2 and p.R617Q SEMA3A, and his father (I:1), carrying only p.Q256K CCDC141, were unaffected. And his sister (II:3), with the p.Q256K CCDC141 and p.R270H PROKR2 mutations, showed reduced armpit and pubic hair, but normal puberty and fertility (Fig. 3 and Table 3).

In Family 4, the proband (II:5) inherited the p.Q409X CCDC141 variant from his normal mother. Family analysis found that his sister (II:2) manifested delayed puberty and two of his nieces (III:2 and III:3) showed dental agenesis. In Family 5, the proband (II:1) inherited two maternal variants of $p . Q 409 X$ CCDC141 and p.Q174R GNRHR, as well as a paternal variant of p.Q65X GNRHR. Both his father and mother showed normal puberty.

In Family 8, the proband (III:9) carried a de novo p.Q871X CCDC141 variant and inherited a p.L80V SOX10 mutation from his mother. He showed bilateral cryptorchidism, hypopigmentation, a white forelock, and 


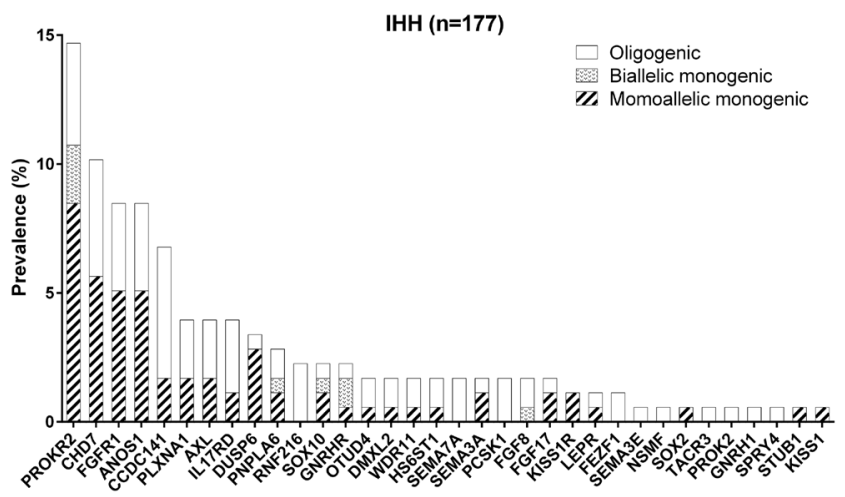

Figure 1

Prevalence of deleterious rare mutations in $34 \mathrm{CHH}$-associated genes in this cohort.

mild hearing loss in addition to KS. His mother (II:11) and maternal grandma (I:2), both carrying the p.L80V SOX10 mutation, showed hypopigmentation, a white forelock and anosmia, but had normal reproductive function and hearing. The maternal uncle (II:1) showed hypogonadism, but with self-reported normal smell. It is a pity we could not determine his genotype. Additionally, two maternal cousins (III:4 and III:6) showed normal puberty and smell, but displayed repeated stillbirth. It seemed that p.L80V SOX10 may lead to various reproductive and nonreproductive pathologies and that CCDC141 might play an additive role in reproductive pathologies.

In Family 11, the proband (II:1) carried a de novo p.E670K FGFR1 variant as well as two paternal variants of p.G1488S CCDC141 and p.G24S HS6ST1, manifested KS with delayed mental development. However, his father, carrying two variants of p.G1488S CCDC141 and p.G24S HS6ST1, appeared normal.

\section{Phenotypes in patients with the same CCDC141 mutations}

p.Q409X CCDC141 was seen in three male probands (4, 5 and 6) and two of them (probands 4 and 5) were diagnosed as $\mathrm{nCHH}$, while proband 6 was diagnosed as $\mathrm{KS}$.
These patients showed some similar phenotypes, such as Tanner II pubic hair, small penis $(<3 \mathrm{~cm}$ in length), small testicular volume $(<5 \mathrm{~mL})$, a nearly identical BMI $\left(23-23.9 \mathrm{~kg} / \mathrm{m}^{2}\right)$ and low bone mass. Proband 5 had no additional phenotypes, whereas probands 4 and 6 did. Proband 4 had congenital anhidrosis (displayed as rarely sweating and feeling a needle-like sting when hot), congenital high myopia and dental agenesis with loss of some teeth. Proband 6 suffered from dental agenesis and right cryptorchidism.

Probands 7 and 8, carrying the de novo p.Q871X CCDC141 mutation, had a micropenis, cryptorchidism, no pubic hair and a normal BMI. However, proband 8, who carried a p.L80V SOX10 mutation, displayed hypopigmentation, a white forelock and hearing loss.

p.G1488S CCDC141 was seen in two male probands (10 and 11) and one female proband (12). Probands 10 and 11 were diagnosed as KS and were overweight, with a BMI of $31 \mathrm{~kg} / \mathrm{m}^{2}$ and $34 \mathrm{~kg} / \mathrm{m}^{2}$, respectively. They also had a micropenis and a small testicular volume $(<5$ $\mathrm{mL}$ ). However, proband 10 suffered from congenital high myopia, whereas proband 11 showed right cryptorchidism, delayed mental development and a delay in major motion during their early years. Proband 12 was 20 years of age with a BMI of $24 \mathrm{~kg} / \mathrm{m}^{2}$. She experienced menarche at the age of 17 , followed by irregular and rare menstrual cycles before suffering from hypogonadotropic hypogonadism (HH) at the age of 18. Fortunately, after a cycle of treatment, she displayed a reversal of $\mathrm{HH}$.

\section{Discussion}

In this study, we systematically analysed the CCDC141 RSVs in a large cohort of Chinese CHH patients via WES. Overall, CCDC141 RSVs were significantly enriched in the $\mathrm{CHH}$ cohort vs our ethnically matched controls (6.8\% vs $0.2 \%, P=0.000)$, supporting the involvement of CCDC141 in CHH. However, up to $75 \%$ of CCDC141 RSVs carriers had at least one mutation in other $\mathrm{CHH}-$ associated genes, and the frequency of multiple rare

\section{Figure 2}

Distribution of CCDC141 RSVs identified in our study. Arabic numerals in the

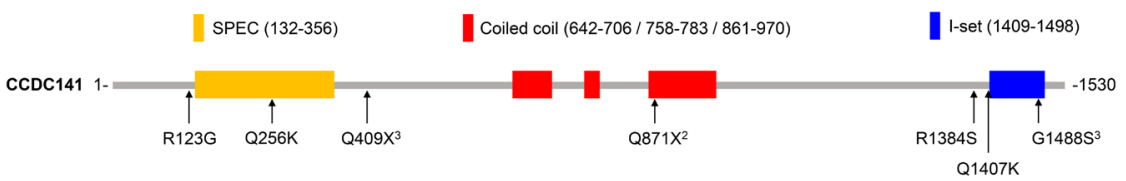
superscript represent the frequency of the mutation in this study. A full colour version of this figure is available at https:// doi.org/10.1530/EJE-19-1018. 

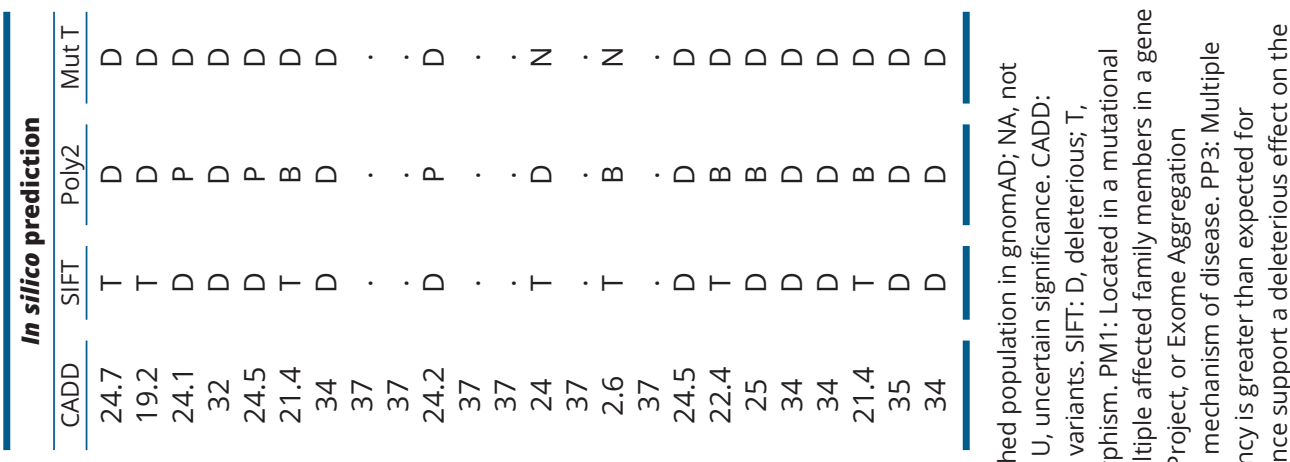

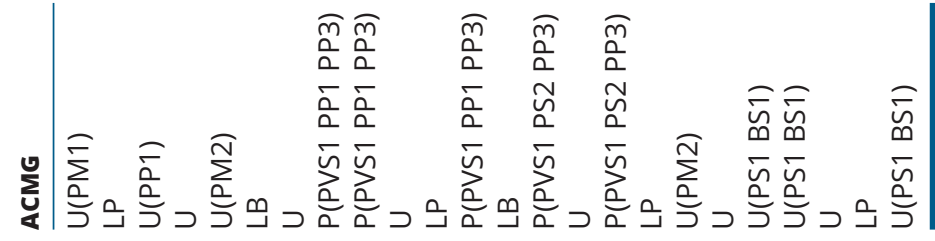


Table 3 Clinical and laboratory findings of the $\mathrm{CHH}$ probands carrying CCDC141 RSVs.

\begin{tabular}{|c|c|c|c|c|c|c|c|c|c|c|}
\hline No. & Sex & Dx & Age & LH & FSH & TS & $\mathbf{E}$ & OB & Bone density & Other phenotypes \\
\hline 1 & M & KS & 23 & 0.6 & 0.57 & 0.1 & 9.7 & Agenesis & low & Right cryptorchidism \\
\hline 2 & $\mathrm{~F}$ & $\mathrm{nCHH}$ & 19 & 0.1 & 0.15 & 1.2 & 20 & Normal & low & Dental agenesis, ataxia \\
\hline 3 & $\mathrm{M}$ & $\mathrm{nCHH}$ & 29 & 1.6 & 0.3 & 0.2 & 10 & Normal & low & Left cryptorchidism, colour weakness \\
\hline 4 & M & $\mathrm{nCHH}$ & 26 & 0.14 & 0.61 & 0.3 & 22 & NA & low & $\begin{array}{l}\text { Dental agenesis, congenital } \\
\text { anhidrosis, high myopia }\end{array}$ \\
\hline 5 & M & $\mathrm{nCHH}$ & 17 & 0.09 & 0.31 & 0.5 & 12.6 & Normal & low & \\
\hline 6 & M & KS & 33 & 0.09 & 0.1 & 0.2 & 12.5 & Hypogenesis & low & Right cryptorchidism \\
\hline 7 & M & KS & 23 & 0.1 & 0.1 & 0.5 & 13 & Agenesis & low & \\
\hline 8 & $\mathrm{M}$ & KS & 19 & 0.14 & 0.1 & 0.1 & 12 & Agenesis & osteoporosis & $\begin{array}{l}\text { Bilateral cryptorchidism, HL } \\
\text { hypopigmentation }\end{array}$ \\
\hline 9 & M & KS & 26 & 0.83 & 0.12 & 0.2 & 11 & NA & osteoporosis & Right cryptorchidism \\
\hline 10 & $\mathrm{M}$ & KS & 19 & 0.6 & 0.56 & 0.8 & 15 & Agenesis & low & Congenital high myopia \\
\hline 11 & $\mathrm{M}$ & KS & 19 & 0.34 & 0.12 & 0.3 & 12 & NA & low & $\begin{array}{l}\text { Dental agenesis, delayed mental } \\
\text { development, cryptorchidism }\end{array}$ \\
\hline 12 & $F$ & $\mathrm{nCHH}$ & 18 & 0.6 & 0.45 & 1.1 & 21 & NA & low & \\
\hline
\end{tabular}

Age means the age of diagnosis. Normal adult men reference range for testosterone (TS): $1.75-7.81 \mathrm{ng} / \mathrm{mL}$, for Estradiol (E2): $<53 \mathrm{pg} / \mathrm{mL}$, for luteinizing hormone (LH): 1.2-8.6 mIU/mL, and for follicle-stimulating hormone (FSH): 1.3-19.3 mIU/mL. Normal adult female reference range for TS: 0.1-1.5 ng/mL, for E2: $31-136 \mathrm{pg} / \mathrm{mL}$, for LH: 1.9-12.5 mIU/L, and FSH 1.3-11 mIU/L.

Dx, diagnosis; F, Female; HL, hearing loss; KS, Kallmann syndrome; M, Male; NA, not available; $\mathrm{nCHH}$, normosmic Idiopathic hypogonadotropic hypogonadism; OB, olfactory bulbs.

variants in one patient was significantly higher in the CCDC141 RSVs' positive group relative to the CCDC141 RSVs' negative group. Moreover, the co-segregation analysis for the eight available $\mathrm{CHH}$ families showed that $75 \%(6 / 8)$ of CCDC141 RSVs were inherited from unaffected parents. In sum, our results confirm the findings from the pioneering investigation by Hutchins et al. (18) reporting that inactivating CCDC141 variants at least partially contributes to $\mathrm{CHH}$.

For some probands, mutations in other $\mathrm{CHH}$ associated genes seemed sufficient to induce $\mathrm{CHH}$, so the simultaneous CCDC141 RSVs might only be 'transient mutations' that have no role in the phenotypes. For instance, proband 7 carried a de novo p.V560I ANOS1 mutation, which alone may be sufficient to cause $\mathrm{CHH}$ in an X-chromosome-linked manner (26). Proband 8 carried a p.L80V SOX10 mutation, which may lead to KS with autosomal dominant inheritance $(27,28)$. Indeed, a panel of SOX10-associated phenotypes, such as hypopigmentation, a white forelock and anosmia were segregated along with the SOX10 mutation in this family. In addition, proband 5 carried a p.Q409X CCDC141 mutation and compound heterozygous GNRHR mutations, which may have been sufficient to cause CHH (9). Interestingly, his mother, carrying two variants of p.Q409X CCDC141 and p.Q174R GNRHR, appeared normal. Proband 11 inherited p.G1488S CCDC141 and p.G24S HS6ST1 from his unaffected father and also carried a de novo p.E670K FGFR1 mutation, which may have been enough to result in $\mathrm{CHH}(29,30)$.
In some circumstances, CCDC141 seems to act synergistically with other $\mathrm{CHH}$-associated genes to cause $\mathrm{CHH}$. For instance, the KS phenotype of proband 1 (III:7 in Family 1) may be the synergistic effect of p.N355I DUSP6 and p.Q1407K CCDC141, as his father (II:5), with $p . Q 1407 K$ CCDC141, and brother (III:8), with p.N355I DUSP6, showed normal puberty. Interestingly, seven individuals in this pedigree showed variations in anosmia; however, we were not able to elucidate the genotypes for most due to their refusal. Proband 2 (III:2 in Family 2), carrying p.R123G CCDC141 and p.V162I PCSK1, manifested dental agenesis, ataxia in infancy and $\mathrm{nCHH}$. Curiously, individuals who only carried one of these variants (II:4, II:7, II:8 with p.R123G CCDC141; II:5, II:10 with p.V162I PCSK1) had normal puberty (Fig. 2 and Table 3). Of note, the proband's maternal grandma (I:4), who also carried these two variants, manifested delayed puberty and no armpit or pubic hair, but showed normal fertility. It thus seems that delayed/absent puberty are consequences from mutations in both CCDC141 and PCSK1. Proband 3 (II:2 in Family 3), with three mutations (p.Q256K CCDC141, p.R270H PROKR2 and p.R617Q SEMA3A), had KS with cryptorchidism and colour weakness. However, individuals who only carried one or two mutations (I:2, II:3, I:1) had normal puberty and fertility. In this circumstance, it seems that these three mutations are necessary for the pathogenicity of $\mathrm{CHH}$, though we cannot rule out the possible contribution of other unidentified genes or environmental factors to the $\mathrm{CHH}$ phenotypes in these pedigrees. 

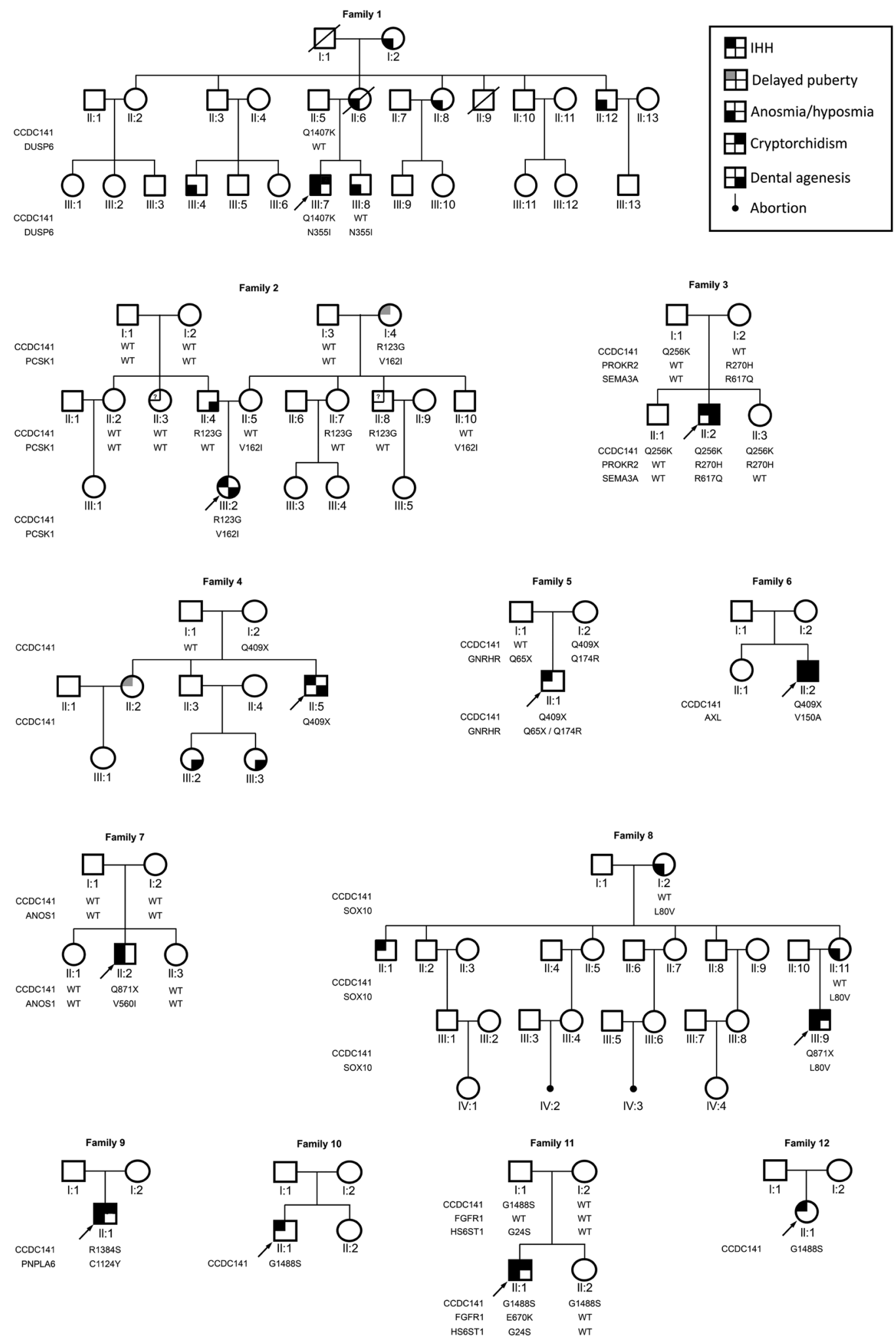

\section{Figure 3}

The $\mathrm{CHH}$ pedigrees with mutations in CCDC141. Subjects in the family are identified by the Roman and Arabic numerals below the symbol, in which the Roman numerals denote the generations. Symbols with a diagonal line = deceased subjects; squares = male; circles $=$ female; arrow $=$ the proband. 
Patients who carried monogenic CCDC141 RSVs showed some phenotypes that were unrelated to those known to pertain to the CCDC141 mutant, such as dental agenesis for proband 4 and anosmia for proband 10, implying that they may carry mutations in other yet unidentified $\mathrm{CHH}$-related gene(s). Actually, in the previous study, oligogenic inheritance/locus heterogeneity and spontaneous clinical reversals were commonly seen in patients with CCDC141 RSVs (18). Therefore, our data reinforce the notion that CCDC141 RSVs may be implicated in the 'second hits' to $\mathrm{CHH}$ and account for the variable expressivity and incomplete penetrance of $\mathrm{CHH}$ in some patients.

CCDC141, a coiled-coil domain-containing protein, functions as a cross-linker between Myosin II and the disrupted-in-schizophrenia 1 (DISC1)centrosome complex. A malfunctioning $C c d c 141$ may lead to neurological and psychiatric disorders $(15,31)$. Recently, Yanagi et al. reported that CCDC141-deficient mice exhibited delayed radial migration with aberrant neural circuit formation, as well as repetitive behaviours, hyperactivity and social abnormalities typically observed in autism spectrum disorder (32). In this cohort, proband 2 was affected with ataxia in infancy, proband 4 manifested congenital anhidrosis and proband 11 showed delayed mental development. However, all patients with CCDC141 RSVs in the previous study were otherwise healthy with normal anthropometric measurements (18), so our study greatly broadens the phenotypic spectrum of CCDC141 more than originally anticipated.

Interestingly, some patients with CCDC141 RSVs also showed vision-related symptoms. Probands 4 and 10 presented with congenital high myopia, and both proband 3 and the CCDC141 RSVs' positive father of proband 2 had colour weakness. It will, therefore, be intriguing to investigate potential roles of CCDC141 in vision development.

\section{Supplementary materials}

This is linked to the online version of the paper at https://doi.org/10.1530/ EJE-19-1018.

\section{Declaration of interest}

The authors declare that there is no conflict of interest that could be perceived as prejudicing the impartiality of this study.

\section{Funding}

This project is financially supported by National Natural Science Foundation of China (81770780, 81728013, 31972913 and 81900948), the Key Research and Development Programs from Hunan Province (2018DK2010, 2018DK2013), Training Program for Excellent Young Innovators of Changsha (kq1802019), Natural Science Foundation of Hunan Province (2018JJ3573) and Foundation of Education Bureau of Hunan Province(18A493), Strategic Priority Research Program of Central South University (ZLXD2017004), and Guangdong Key Project in 'Development of new tools for diagnosis and treatment of Autism' (2018B030335001).

\section{Acknowledgements}

The authors would like to thank the patients for their participation in the study as well as their family members for their collaboration.

\section{References}

1 Kim SH. Congenital hypogonadotropic hypogonadism and Kallmann Syndrome: past, present, and future. Endocrinology and Metabolism 201530 456-466. (https://doi.org/10.3803/EnM.2015.30.4.456)

2 Quinton R, Duke VM, Robertson A, Kirk JM, Matfin G, de Zoysa PA, Azcona C, MacColl GS, Jacobs HS, Conway GS et al. Congenital gonadotrophin deficiency: genetic questions addressed through phenotypic characterization. Clinical Endocrinology 200155 163-174. (https://doi.org/10.1046/j.1365-2265.2001.01277.x)

3 Costa-Barbosa FA, Balasubramanian R, Keefe KW, Shaw ND, Al-Tassan N, Plummer L, Dwyer AA, Buck CL, Choi JH, Seminara SB et al. Prioritizing genetic testing in patients with Kallmann syndrome using clinical phenotypes. Journal of Clinical Endocrinology and Metabolism 201398 E943-E953. (https://doi.org/10.1210/jc.20124116)

4 Sandhya N, Swati J, Anurag L, Varsha J, Amol B, Reshma P, Alka E, Padma M, Tushar B, Nalini S et al. Spectrum of phenotype and genotype of congenital isolated hypogonadotropic hypogonadism in Asian Indians. Clinical Endocrinology 201685 100-109. (https://doi. org/10.1111/cen.13009)

5 Bonomi M, Vezzoli V, Krausz C, Guizzardi F, Vezzani S, Simoni M, Bassi I, Duminuco P, Di Iorgi N, Giavoli C et al. Characteristics of a nationwide cohort of patients presenting with isolated hypogonadotropic hypogonadism (IHH). European Journal of Endocrinology 2018178 23-32. (https://doi.org/10.1530/EJE-17-0065)

6 Casoni F, Malone SA, Belle M, Luzzati F, Collier F, Allet C, Hrabovszky E, Rasika S, Prevot V, Chédotal A et al. Development of the neurons controlling fertility in humans: new insights from 3D imaging and transparent fetal brains. Development 2016143 3969-3981. (https://doi.org/10.1242/dev.139444)

7 Pitteloud N, Durrani S, Raivio T \& Sykiotis GP. Complex genetics in idiopathic hypogonadotropic hypogonadism. Frontiers in Hormone Research 201039 142-153. (https://doi.org/10.1159/000312700)

8 Miraoui H, Dwyer AA, Sykiotis GP, Plummer L, Chung W, Feng B, Beenken A, Clarke J, Pers TH, Pitteloud N et al. Mutations in FGF17, IL17RD, DUSP6, SPRY4, and FLRT3 are identified in individuals with congenital hypogonadotropic hypogonadism. American Journal of Human Genetics 201392 725-743. (https://doi.org/10.1016/j. ajhg.2013.04.008)

9 Gonçalves CI, Aragüés JM, Bastos M, Barros L, Vicente N, Carvalho D \& Lemos MC. GNRHR biallelic and digenic mutations in patients with normosmic congenital hypogonadotropic hypogonadism. Endocrine Connections 20176 360-366. (https://doi.org/10.1530/EC-17-0104)

10 Amato L, Montenegro LR, Lerario AM, Jorge A, Guerra Junior G, Schnoll C, Renck AC, Trarbach EB, Costa E, Mendonca BB et al. New genetic findings in a large cohort of congenital hypogonadotropic hypogonadism. European Journal of Endocrinology 2019181 103-119. (https://doi.org/10.1530/EJE-18-0764)

11 Young J, Bouligand J, Francou B, Raffin-Sanson ML, Gaillez S, Jeanpierre M, Grynberg M, Kamenicky P, Chanson P, Brailly-Tabard S 
et al. TAC3 and TACR3 defects cause hypothalamic congenital hypogonadotropic hypogonadism in humans. Journal of Clinical Endocrinology and Metabolism 201095 2287-2295. (https://doi. org/10.1210/jc.2009-2600)

12 Ke R, Ma X \& Lee L. Understanding the functions of kisspeptin and kisspeptin receptor (Kiss1R) from clinical case studies. Peptides 2019 120 170019. (https://doi.org/10.1016/j.peptides.2018.09.007)

13 Zhao Y, Wu J, Wang X, Jia H, Chen DN \& Li JD. Prokineticins and their $\mathrm{G}$ protein-coupled receptors in health and disease. Progress in Molecular Biology and Translational Science 2019161 149-179. (https://doi.org/10.1016/bs.pmbts.2018.09.006)

14 Giacobini P, Kopin AS, Beart PM, Mercer LD, Fasolo A \& Wray S. Cholecystokinin modulates migration of gonadotropin-releasing hormone-1 neurons. Journal of Neuroscience 200424 4737-4748. (https://doi.org/10.1523/JNEUROSCI.0649-04.2004)

15 Solecki DJ, Trivedi N, Govek EE, Kerekes RA, Gleason SS \& Hatten ME. Myosin II motors and F-actin dynamics drive the coordinated movement of the centrosome and soma during CNS glial-guided neuronal migration. Neuron 200963 63-80. (https://doi. org/10.1016/j.neuron.2009.05.028)

16 Hutchins BI \& Wray S. Capture of microtubule plus-ends at the actin cortex promotes axophilic neuronal migration by enhancing microtubule tension in the leading process. Frontiers in Cellular Neuroscience 20148 400. (https://doi.org/10.3389/fncel.2014.00400)

17 Hutchins BI, Kotan LD, Taylor-Burds C, Ozkan Y, Cheng PJ, Gurbuz F, Tiong JD, Mengen E, Topaloglu AK, Wray S et al. CCDC141 mutation identified in anosmic hypogonadotropic hypogonadism (Kallmann syndrome) alters GnRH neuronal migration. Endocrinology 2016157 1956-1966. (https://doi.org/10.1210/en.2015-1846)

18 Turan I, Hutchins BI, Hacihamdioglu B, Kotan LD, Gurbuz F, Ulubay A, Mengen E, Yuksel B, Wray S \& Topaloglu AK. CCDC141 mutations in congenital hypogonadotropic hypogonadism. Journal of Clinical Endocrinology and Metabolism 2017102 1816-1825. (https:// doi.org/10.1210/jc.2016-3391)

19 Guo H, Jin X, Zhu T, Wang T, Tong P, Tian L, Peng Y, Sun L, Wan A, Chen $\mathrm{J}$ et al. SLC39A5 mutations interfering with the BMP/TGF- $\beta$ pathway in non-syndromic high myopia. Journal of Medical Genetics 201451 518-525. (https://doi.org/10.1136/jmedgenet-2014-102351)

20 Men M, Li W, Chen H, Wu J, Feng Y, Guo H \& Li JD. Identification of a novel CNV at 8q13 in a family with Branchio-Oto-Renal syndrome and epilepsy. Laryngoscope 2020130 526-532. (https://doi. org/10.1002/lary.27941)

21 Shaw ND, Seminara SB, Au MG, Plummer L, Martin KA, Quinton R, Mericq V, Crowley Jr WF, Pitteloud N, Hall JE et al. Expanding the phenotype and genotype of female GnRH deficiency. Journal of Clinical Endocrinology and Metabolism 201196 E566-E576. (https:// doi.org/10.1210/jc.2010-2292)

22 Stamou MI, Cox KH \& Crowley WF. Discovering genes essential to the hypothalamic regulation of human reproduction using a human disease model: adjusting to life in the '-Omics' era. Endocrine Reviews 201536 603-621. (https://doi.org/10.1210/er.2015-1045)
23 Maione L, Dwyer AA, Francou B, Guiochon-Mantel A, Binart N, Bouligand J \& Young J. Genetics in endocrinology: Genetic counseling for congenital hypogonadotropic hypogonadism and Kallmann syndrome: new challenges in the era of oligogenism and next-generation sequencing. European Journal of Endocrinology 2018 178 R55-R80. (https://doi.org/10.1530/EJE-17-0749)

24 den Dunnen JT \& Antonarakis SE. Mutation nomenclature extensions and suggestions to describe complex mutations: a discussion. Human Mutation 200015 7-12. (https://doi.org/10.1002/ (SICI) 1098-1004(200001)15:1<7::AID-HUMU4>3.0.CO;2-N)

25 Richards S, Aziz N, Bale S, Bick D, Das S, Gastier-Foster J, Grody WW, Hegde M, Voelkerding K, Rehm HL \& ACMG Laboratory Quality Assurance Committee. Standards and guidelines for the interpretation of sequence variants: a joint consensus recommendation of the American College of Medical Genetics and Genomics and the Association for Molecular Pathology. Genetics in Medicine 201517 405-424. (https://doi.org/10.1038/gim.2015.30)

26 Sato N, Katsumata N, Kagami M, Hasegawa T, Hori N, Kawakita S, Minowada S, Shimotsuka A, Shishiba Y, Yokozawa M et al. Clinical assessment and mutation analysis of Kallmann syndrome 1 (KAL1) and fibroblast growth factor receptor 1 (FGFR1, or KAL2) in five families and 18 sporadic patients. Journal of Clinical Endocrinology and Metabolism 200489 1079-1088. (https://doi.org/10.1210/jc.2003030476)

27 Pingault V, Bodereau V, Baral V, Marcos S, Watanabe Y, Chaoui A, Fouveaut C, Leroy C, Vériermine O \& Francannet C. Loss-of-function mutations in SOX10 cause Kallmann syndrome with deafness. American Journal of Human Genetics 201392 707-724. (https://doi. org/10.1016/j.ajhg.2013.03.024)

28 Dai W, Wu J, Zhao Y, Jiang F, Zheng R, Chen DN, Men M \& Li JD. Functional analysis of SOX10 mutations identified in Chinese patients with Kallmann syndrome. Gene 2019702 99-106. (https:// doi.org/10.1016/j.gene.2019.03.039)

29 Men M, Wu J, Zhao Y, Xing X, Jiang F, Zheng R \& Li JD. Genotypic and phenotypic spectra of FGFR1, FGF8, and FGF17 mutations in a Chinese cohort with isolated hypogonadotropic hypogonadism. Fertility and Sterility 2020113 158-166. (https://doi.org/10.1016/j. fertnstert.2019.08.069)

30 Dodé C, Levilliers J, Dupont JM, De AP, Le N, Soussiyanicostas N, Coimbra RS, Delmaghani S, Compainnouaille S \& Baverel F. Loss-of-function mutations in FGFR1 cause autosomal dominant Kallmann syndrome. Nature Genetics 200333 463-465. (https://doi. org/10.1038/ng1122)

31 Fukuda T, Sugita S, Inatome R \& Yanagi S. CAMDI, a novel disrupted in schizophrenia 1 (DISC1)-binding protein, is required for radial migration. Journal of Biological Chemistry 2010285 40554-40561. (https://doi.org/10.1074/jbc.M110.179481)

32 Fukuda T, Nagashima S, Abe T, Kiyonari H, Inatome R \& Yanagi S. Rescue of CAMDI deletion-induced delayed radial migration and psychiatric behaviors by HDAC6 inhibitor. EMBO Reports 201617 1785-1798. (https://doi.org/10.15252/embr.201642416)

Received 14 December 2019

Revised version received 9 May 2020

Accepted 8 June 2020 